\title{
Correction to: DSMC Evaluation Stages: Fostering Robust and Safe Behavior in Deep Reinforcement Learning
}

Timo P. Gros, Daniel Höller, Jörg Hoffmann, Michaela Klauck, Hendrik Meerkamp, and Verena Wolf

\author{
Correction to: \\ Chapter "DSMC Evaluation Stages: Fostering Robust \\ and Safe Behavior in Deep Reinforcement Learning" \\ in: A. Abate and A. Marin (Eds.): Quantitative Evaluation \\ of Systems, LNCS 12846, \\ https://doi.org/10.1007/978-3-030-85172-9_11
}

In an older version of this paper, there was a mistake in line 12 of the algorithm on page 206. This was corrected. 